\title{
OSLo: Optical Sensor Localization through Mesh Networked Cameras
}

\author{
Hassaan Janjua*, Fan Yang*, Mahmoud Ammar*, David Newton ${ }^{\dagger}$, Seonhi Ro ${ }^{\dagger}$, Sam Michiels*, Danny Hughes* \\ *imec-DistriNet, KU Leuven, Belgium \\ $\{$ first name\}.\{last name\}@cs.kuleuven.be \\ ${ }^{\dagger}$ Ford Motor Company, Germany \\ \{first name first letter\}\{last name $\} @$ ford.com
}

\begin{abstract}
Accurate indoor positioning remains an open research question. Existing solutions are either expensive, shortrange, or inaccurate. A new approach is therefore required to cost-effectively and accurately support localization in large indoor environments. We tackle this problem by introducing a practical optical localization scheme, called OSLo, that costeffectively scales to support large buildings. OSLo uses a meshed network of low-cost cameras as localization anchors and smart LEDs as tags, which transmit their IDs and context sensor data over the meshed cameras using optical communication. OSLo is capable of localizing dense deployments of tags with an accuracy of under 1 meter at a distance of 35 meters from the localization anchor. Furthermore, smart LED tags can be manufactured for less than \$1. We systematically evaluate the performance of OSLo in the context of a real-world car localization use-case at Ford Motor Company in Germany and demonstrate promising results in terms of detection distance, and localization accuracy.

Index Terms-localization, indoor localization, visible light communication, mesh network
\end{abstract}

\section{INTRODUCTION}

Indoor localization is a large and growing market [1], which enables applications such as Industry 4.0, smart parking, and Automated Guided Vehicles (AGVs). At its core, an indoor localization system requires three basic operations; (i.) identification of the tag which is to be localized, (ii.) localization of tags across a number of sub-regions, and (iii.) integration of identity and location data. An ideal indoor localisation system minimises cost and complexity by offering long range, lowcost tags, long battery life and high accuracy.

Internet of Things (IoT) technologies combined with Radio Frequency (RF) [2]-[5], Ultra Wide Band (UWB) [6], acoustic ranging [7], or optical localization [8]-[12] enable the vision of low-cost and easy-to-deploy indoor localization systems with long battery lifetimes. Despite this promising vision, contemporary localization systems are either inaccurate, expensive, or power-hungry.

Optical localisation [8]-[12] has emerged as particularly promising localisation technology, with high accuracy and low implementation complexity. However, current optical localization solutions rely on a dense infrastructure of smart LEDs as localization anchors, increasing infrastructure costs and deployment complexity. While the localization tags are either required to have a dedicated camera or photo diode based custom hardware. These characteristics are particularly problematic in applications that demand localization of multiple objects in large indoor industrial environments such as factories and warehouses. We discus existing optical localization technologies in Section II. Industrial localization scenarios require the localization tag to be extremely low cost and battery life needs to be in the order of months. Additional requirements include: sub-meter accuracy, minimal setup requirements and long-range detection. To the best our knowledge, none of the existing localization systems meet these requirements. In contrast to prior work on optical localization [8]-[12], we propose to address this problem through the introduction of OSLo, a novel optical localization scheme that delivers accurate, long-life, and low-cost indoor localization through meshed networks of wireless infrared (IR) cameras.

OSLo makes the following unique contributions: (i) a novel optical localization technique with more than twice the tagto-anchor range of previously proposed schemes, (ii) an extremely low-cost battery powered localization tag that enables large-scale cost-effective industrial deployments and (iii) mesh networked localization anchors that work together to present a unified view of localization tags for large indoor areas.

Critically, OSLo preserves user privacy by firstly operating outside of the visible light spectrum and secondly by performing all image analysis on the anchors at the edge of the network, rather than transmitting information to the cloud for analysis.

We evaluate OSLo in an industrial indoor parking setting where the objects to be located (cars, awaiting retrofit) are placed for longer periods of time in a relatively large indoor space. This scenario requires extremely low-cost localization tags as the volume of the objects to be located is large (over $1 \mathrm{~m}$ cars per year) and the cost of the localization tag is therefore critical to minimizing the total cost of ownership for the system. Fast localization is not required in this setting, as cars in the warehouses are placed there for multiple hours before retrofit is concluded.

The remainder of this paper is structured as follows. Section II provides background on optical localization technologies. Section III describes the design of the proposed localization system. Section IV provides the implementation details of the prototype indoor localization system. Section V evaluates OSLo. Section VI compares OSLo to related camera-based localization technologies. Finally, Section VII concludes and 
discusses directions for future work.

\section{BACKGROUND}

A typical Optical indoor localization system consists of LED luminaires, installed primarily for illumination, which are modified to serve as localization anchors that transmit their location information via VLC [13]. The localization tag can be a single Photo Diode (PD), an array of PDs, or a camera. The location signal is typically encoded in a modulated signal that is too fast to be visible to the human eye, while localization is achieved using one of RSS, ToF, TDoA, or AoA techniques. More recently an inverted approach is being explored by Saska et al. [12] to utilize Ultra Violet (UV) LEDs as the localization tag and a camera as the localization anchor.

Li et al. [10] used light received signal strength indication as a measure of distance for optical localization. As with radiobased schemes, the RSSI of light is inversely proportional to the square of the distance between the transmitter and receiver, modified by the irradiation angle and the incidence angle. This relationship enables localization using either model- or fingerprint-based RSSI of light techniques. Both approaches give promising results with an accuracy of up to $0.4 \mathrm{~m}$ where the distance between the transmitter and the receiver is not more than $3 \mathrm{~m}$ [10]. Jung et al. [14] utilize a series of photodetectors to measure TDoA of signal from multiple LEDs, the TDoA is used to localize the tag. The inaccuracy for the TDoA based localization was shown to be less than $1 \mathrm{~cm}$ in a $5 \mathrm{~m} \times 5 \mathrm{~m} \times 3 \mathrm{~m}$ area. AoA-based localization using VLC typically requires an array of PDs. Lee et al. [15] used a circular array of uniformly placed PDs in the tag. The angle of arrival is estimated using the difference in light intensity among the PDs and the position of the receiving PDs. PDbased localization schemes have high accuracy but very limited range from the localization anchors. This requires a dense localization anchor deployment, increasing the overall cost of the localization system. Such solutions are not feasible for large indoor environments.

TABLE I: Optical Localization

\begin{tabular}{|llllll|}
\hline & & & & \multicolumn{2}{c|}{ Accuracy } \\
& & Multi-tag & Range & Meter & Degrees \\
\hline RSSI & {$[10]$} & Yes & 5 & 0.8 & 9.09 \\
TDoA & {$[14]$} & Yes & $5 \times 5 \times 3$ & 0.01 & 0.11 \\
AoA & {$[15]$} & Yes & $5 \times 5 \times 3$ & 0.3 & 3.43 \\
Camera & {$[16]$} & Yes & 0.9 & 0.2 & 12.53 \\
Camera & {$[17]$} & Yes & 3 & 0.05 & 0.95 \\
Camera & {$[12]$} & No & 15 & N/A & 1.15 \\
Camera & {$[8]$} & Yes & 2.8 & 0.15 & 1 \\
Camera & OSLo & Yes & 35 & 1 & 1.64 \\
\hline
\end{tabular}

Ganick et al. [18] used a single camera as the localization tag when there are three or more LEDs in the view. The technique uses triangulation to calculate the position of the localization tag. Nakazawa et al. [16] used a similar triangulation technique in combination with a fish-eye lens, which increases the field of view of the camera. The fisheye lens introduces radial distortion to the image, which may be removed by modeling the radial distortion using intrinsic camera calibration as described by Komagata et al. [19]. Tanaka et al. [17] used an accelerometer to determine camera orientation along three axes and requires only two LEDs to determine location. Pengjin Xie et al. [8] developed a lowcost localization system that utilizes a chip-less and batteryless optical tag which exhibits different colors when viewed from a different angle using a combination of birefringent and polarizer materials. tags created in such a manner are extremely low cost but localization using these tags is limited to only 2.8 meters.

Table I summarizes the localization accuracy of optical localization techniques. We can observe from Table I that Optical localization provides better accuracy as compared to other techniques. However, in most of the existing solutions, the range of localization is limited to a few meters. The limited range translates to dense localization anchor deployment that in turn increases the complexity and cost of the overall system. In contrast, OSLo presents a long range localization system with high localization accuracy.

\section{A. Requirements}

Based upon the overview of existing localisation schemes presented above, and our real-world case study at Ford Motor Company, we identify the following requirements for the design of OSLo:

1) Minimal tag cost, taking into account that in most scenarios there are likely to be far more tags than anchors (thousands of times more in our car localisation use-case).

2) Long localization range between the tags and the anchors to enable cost-effective coverage of large indoor areas.

3) Sub-meter localization accuracy to distinguish between densely co-located objects.

4) Long battery life to minimize maintenance costs due to battery replacement.

5) Privacy preservation to ensure that the optical localization system cannot be used to track humans working in the environment.

The following section describes the design of OSLo, an optical localisation system which aims to address these requirements.

\section{DESIGN OF OSLO}

The OSLo localization system consists of mesh networked cameras that collaborate to provide a unified view of the localization tags on a map of the target area. An embedded processor is attached to each camera that extracts the image coordinates and ID of tags within the camera's field of view and then transmits this information via the mesh network to a back-end server, which applies the localization algorithm to locate the tags and stores this information in a database. We refer to the combination of the camera and the embedded processor as an optical sensor. OSLo utilizes two algorithms for localization; a single camera localization algorithm, which is used when the height of the localization tag is available 
(Section III-B) and a multi-camera algorithm, which is used when the localization tag's height is unknown (Section III-C). The localization tag is a sub-\$1 IR LED-based battery-powered device that can last up to a year on a single set of batteries. The LED tag transmits its ID periodically using an optical communication protocol, the details of which are presented in section III-D.

The optical sensors are deployed at an inclined angle so that the camera view covers more target area as shown in Figure 1. It should be noted that the only information exported from the optical sensor is the image coordinates and ID of the localization tags. This enables OSLo to be deployed in privacysensitive environments. Once a tag is identified, the optical sensor transmits the image coordinates and the ID information to the back-end server, which applies the localization algorithm and sends the location information for storage in a database. The OSLo visualization system connects to this database and displays the location tags on a map.

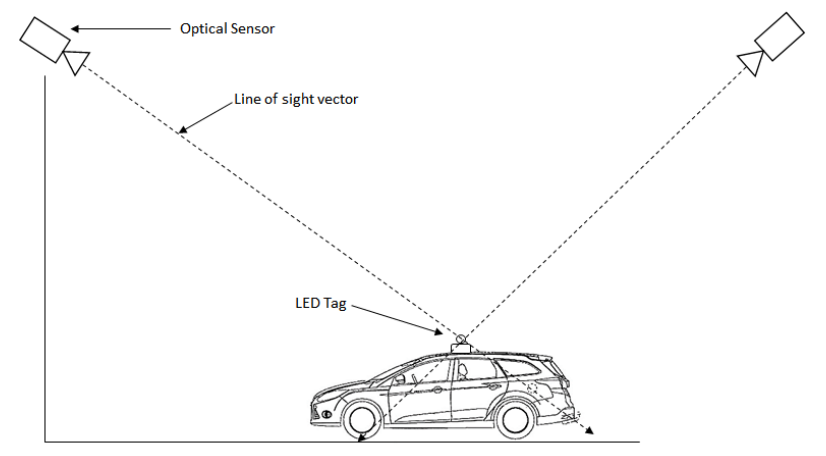

Fig. 1: Visualisation of Optical Localisation

\section{A. Localization Pipeline}

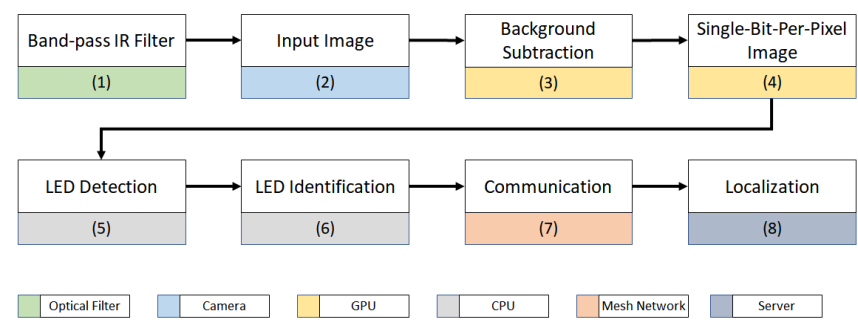

Fig. 2: Localization Pipeline

The input from the camera is processed through a pipeline shown in Figure 2. In step (1), the band-pass IR filter performs the first part of image processing by removing light sources other than the $940 \mathrm{~nm}$ wavelength range (+/$10 \mathrm{~nm})$. The filtered image is captured by the camera and is available for further processing, depicted as input image in the localization pipeline. The two operations in steps (3) and (4) are performed in the Graphics Processing Unit (GPU) that reads the image from the camera, performs background subtraction and converts it in a single bit per pixel format.
The single bit per pixel conversion is important for achieving a fast processing rate. Normally, an image is captured in a 4 byte per pixel format but the LED identification algorithm only requires a boolean value per pixel after the background subtraction. The conversion to a single bit per pixel reduces the image size by a factor of 32 . The single bit per pixel format enables further optimization during the LED detection operation. After the background subtraction and the single bit per pixel conversion, we are left with an image that only has 1 pixels at the LED positions, while the rest of the image is filled with 0s. We use a modified flood-fill [20] algorithm to find contours created by the LED in the image, shown as step (4). Since most of the pixels are 0 in this image and each 4byte word contains information of 32 pixels, we optimize the detection algorithm to check the word for non-zero values first. The contour detection algorithm returns a list of contours in the current image with each contour represented by its centroid. The next step (6) operates on all detected contours to check if the contours represent an LED and then extract its ID. This operation spans multiple frames while keeping track of the detected contours by their centroid. Once a LED is verified, its ID is extracted from the pattern accumulated over multiple frames. After the LED identification, the pixel coordinates of the LED, along with its ID are communicated to the backend server (step 7). In step (8) one of the two localization algorithm calculates the world coordinates of the LED.

\section{B. Single Camera Based Localization}

An image is a projection of the $3 \mathrm{D}$ space on a $2 \mathrm{D}$ surface. This transformation loses the distance information of the projected points. Thus, each pixel on the image represents a line of sight vector originating from the center of projection. The image projection is modeled as a pinhole camera model [21] between two coordinates systems with image point denoted as $\left[\begin{array}{lll}x & y & 1\end{array}\right]^{T}$ and the corresponding world point denoted as $\left[\begin{array}{llll}X & Y & Z & 1\end{array}\right]^{T}$. Equation 1 expresses the relationship between the image pixel coordinates $p$ and the world coordinates $P$ of a point.

$$
\left[\begin{array}{l}
x \\
y \\
1
\end{array}\right]=s \cdot M \cdot R \cdot\left[\begin{array}{l}
X \\
Y \\
Z \\
1
\end{array}\right]+T
$$

$\mathrm{M}, \mathrm{R}$, and $\mathrm{T}$ in Equation 1 represent an intrinsic camera calibration matrix, the camera rotation matrix, and camera translation vector respectively. The variable $\mathrm{s}$ is an arbitrary scale factor that expresses the fact that the transformation is defined up to that scale factor. The depth information of the world coordinate is lost during this transformation which makes it impossible to localize the 3D world coordinates of the projection point without further information. Planar homography [21] relates the transformation between two planes. This transformation can be used to calculate a mapping from the image coordinates to a plane in the world coordinate system as explained in [22]. Planar homography assumes that all observed points lie on a fixed plane $\mathrm{Z}=0$. The homography 
matrix for the $\mathrm{Z}=0$ plane can be expressed as Equation 2 [22].

$$
\left[\begin{array}{l}
x \\
y \\
1
\end{array}\right]=s M\left[\begin{array}{lll}
r_{1} & r_{2} & T
\end{array}\right]\left[\begin{array}{l}
X \\
Y \\
1
\end{array}\right]
$$

$r_{1}$, and $r_{2}$, are the first two columns of the camera rotation matrix in Equation 2 while $\mathrm{s}, \mathrm{M}$ and $\mathrm{T}$ are the same as in Equation 1. The simplification of $Z=0$ gives a $3 \times 3$ Homography matrix $\mathrm{H}=\mathrm{sM}\left[\begin{array}{lll}r_{1} & r_{2} & T\end{array}\right]$. The Homography matrix is calculated using a mapping of four or more points between image and world coordinates [22].

$$
\left[\begin{array}{l}
X \\
Y \\
1
\end{array}\right]=H^{-1}\left[\begin{array}{l}
x \\
y \\
1
\end{array}\right]
$$

\section{Arbitrary Plane Homography}

We generalise fixed planar homography, which assumes that all points lie on the plane $\mathrm{Z}=0$ such that all points lie on an arbitrary plane parallel to the $\mathrm{Z}$ plane. This generalization allows us to provide the height of the LED as a parameter to the localization system. As opposed to planar homography, this approach is independent of the height of the localization plane. Equation 4 represents the camera projection model.

$$
\left[\begin{array}{l}
x \\
y \\
1
\end{array}\right]=s M\left(R\left[\begin{array}{l}
X \\
Y \\
Z
\end{array}\right]+T\right)
$$

The inverse project equation can be derived as follows:

$$
\left[\begin{array}{l}
X \\
Y \\
Z
\end{array}\right]=R^{-1}\left(\frac{1}{s} \cdot M^{-1}\left[\begin{array}{l}
x \\
y \\
1
\end{array}\right]-T\right)
$$

After applying the matrix transformations, Equation 5 can be expressed as shown in Equation 6. Where $\mathrm{x}_{0}, \mathrm{y}_{0}$ and $\mathrm{w}_{0}$ represent the column vector values after applying the inverse camera matrix and inverse rotation matrix transformations to the image coordinate vector $\left[\begin{array}{lll}x & y & 1\end{array}\right]$.

$$
\left[\begin{array}{l}
X \\
Y \\
Z
\end{array}\right]=\frac{1}{s} \cdot\left[\begin{array}{l}
x_{0} \\
y_{0} \\
w_{0}
\end{array}\right]-R^{-1} T
$$

In Equation 6, the calculated world coordinates are correct up to a scale factor s. This scale factor also gives us the capability to adjust the scaling to fit an arbitrary value for $Z$. In Equation 7, we provide the height of the LED as $Z_{0}$ and scale the $\mathrm{X}$ and $\mathrm{Y}$ coordinates to match the provided $\mathrm{Z}_{0}$.

$$
\begin{aligned}
s & =\frac{w_{0}}{Z_{0}+T_{3}^{\prime}} \\
X & =\frac{1}{s} x_{0}-T_{1}^{\prime} \\
Y & =\frac{1}{s} y_{0}-T_{2}^{\prime}
\end{aligned}
$$

In the set of Equations $7 \mathrm{~T}^{\prime}=\mathrm{R}^{-1} \mathrm{~T}$ and $\mathrm{T}^{\prime}{ }_{1}, \mathrm{~T}_{2}^{\prime}$ and $\mathrm{T}_{3}{ }_{3}$ are the components of $\mathrm{T}$ ' corresponding to the $\mathrm{X}, \mathrm{Y}$ and $\mathrm{Z}$ coordinates respectively. Applying the scale factor to $\mathrm{X}$ and $\mathrm{Y}$ gives the normalized world coordinates based on the parameterized height. An evaluation of arbitrary plane homography is presented in Section V-C.

\section{Multiple Camera Based Localization}

Equation 5 specifies the relationship between the real world coordinates and the projected coordinates on an image. The information loss due to projection on 2D space from the real world is captured by the scaling factor $\frac{1}{s}$. Hence, if we calculate the $\frac{1}{s}$ parameter, it is possible to calculate the complete inverse projection for a given image coordinate. Since we have the image coordinates from both the cameras for the same 3D point, we can use this relationship to specify the scaling factor for both cameras in terms of the world point as expressed in Equation 8.

$$
\begin{aligned}
{\left[\begin{array}{l}
X \\
Y \\
Z
\end{array}\right] } & =\frac{1}{s_{1}} R_{1}^{-1} M_{1}^{-1}\left[\begin{array}{c}
x_{1} \\
y_{1} \\
1
\end{array}\right]-R_{1}^{-1} T_{1} \\
& =\frac{1}{s_{2}} R_{2}^{-1} M_{2}^{-1}\left[\begin{array}{c}
x_{2} \\
y_{2} \\
1
\end{array}\right]-R_{2}^{-1} T_{2}
\end{aligned}
$$

Combining the two Equations in 8 with two unknowns $\frac{1}{s_{1}}$ and $\frac{1}{s_{2}}$ gives us three equations with two unknowns.

This leads to an over-constraint system. With three equations, one for each coordinate axis. It is possible to have no solution that satisfies all three equations because of the measurement errors. We address this problem by using the least squares method to find the two parameters that gives an approximate solution for both $\frac{1}{s_{1}}$ and $\frac{1}{s_{2}}$. The resulting parameter $\frac{1}{s_{1}}$ and $\frac{1}{s_{2}}$ can be used with the two Equations in 8 to calculate the real world $3 \mathrm{D}$ coordinates of the given world point .

\section{LED to Camera Data Transmission}

In designing a transmission protocol, we kept several considerations in mind. (1) Slow data transmission that is limited by the camera frame rate, (2) the lack of a common clock between the transmitter and receiver, (3) variable drift and (4) the need to filtered out transmission errors.

Considering the low transmission rate, the only information that the LED transmits is its ID and 1-bit battery indicator, which indicates whether the battery needs to be replaced. We use the Manchester encoding system [23] to encode our data on top of an ON/OFF keying technique. The use of Manchester encoding resolves the clock synchronization and drift issues. We use a one-bit preamble to mark the start of transmission. The camera continuously captures and processes the images at a fixed frame-rate. Whenever a difference from the background is detected, the system isolates the target coordinate and starts receiving data from that coordinate. In order to detect 
transmission errors, we use a 4 bit Cyclic Redundancy Check (CRC) for the 16-bits of data. The format of the entire data packet is visualized in Figure 3.

\begin{tabular}{|l|l|l|l|} 
Preamble & \multicolumn{2}{l}{ Battery } \\
Indicator & LED ID & CRC-4 \\
\hline 1 & 1 & 15 & 4 \\
\hline
\end{tabular}

Fig. 3: Packet Structure

\section{E. Optical Sensor Calibration}

We perform the optical sensor calibration in two steps. In the first step, the intrinsic calibration parameters for each of the optical sensors are calculated. The intrinsic calibration is independent of the position of the optical sensor. Therefore, it is calculated offline. In the second step, extrinsic calibration is performed at the deployment position. Extrinsic calibration aims to determine the coordinates and the orientation of the optical sensor with respect to a world origin. This world origin is fixed in a given environment and all localization is performed in its coordinates system that is represented as an offset to the world origin coordinates and orientation. Extrinsic calibration requires manual measurement of reference point coordinates that are provided to the system along with a mapping to the image coordinates. We use the LED detection and identification mechanism to create the mapping between the world coordinates with the image coordinates. We place an LED at each reference point in the field of view of the optical sensor. The world coordinates of the reference points are provided to the system in the form of a tuple (ID, X, Y, $\mathrm{Z}$ ), where ID is the given ID of the LED and $\mathrm{X}, \mathrm{Y}$, and $\mathrm{Z}$ are the coordinates of the LED with respect to a deployment world origin. Initially the optical sensors are deployed in a so-called calibration mode. During the calibration mode, an optical sensor keeps looking for the reference point IDs in the environment. As soon as it identifies the required reference points, it creates a mapping between the image coordinate and the provided world coordinates of the reference points based on the IDs of the reference points. This mapping is used to calculate the extrinsic calibration parameters for the optical sensor. Once the extrinsic parameters are calculated, they are stored in the localization system and the optical sensor enters the localization mode. World Origin: The deployment world origin is fixed for deployment so that all optical sensors perform localization in the same coordinate system. The use of a common coordinate system simplifies localization that involves multiple optical sensors.

\section{F. LED Tag Design}

The constraints of our application use-case demand a low localisation tag cost. To achieve a minimal manufacturing cost, the tag hardware is designed to be as simple as possible. A schematic of the design is shown in Figure 4. The tag is designed to be powered by powered by batteries, a dedicated power rail is generated for driving the LED using a buck converter, which regulates the supplied voltage appropriately. The microcontroller unit (MCU) transmits data by switching the LED on and off.

The tag minimizes its power consumption through aggressive duty-cycling, and remaining in sleep mode as long as possible. The tag consumes $2 \mu \mathrm{A}$ when the MCU disables the LED power rail and enters deep sleep mode. Upon the expiration of the periodic timer, the MCU wakes up and enters transmission mode, returning to sleep immediately thereafter. During localisation, the tag consumes an average of $270 \mathrm{~mA}$.

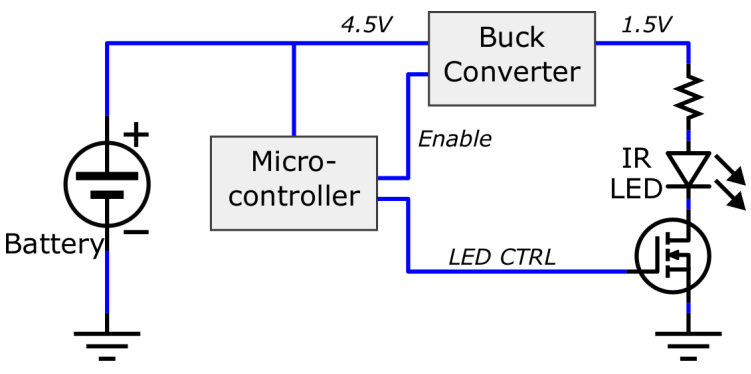

Fig. 4: LED Tag Circuit Diagram.

\section{IMPLEMENTATION}

We have implemented and deployed OSLo in an industrial setting at the Ford company in Germany. In our development scenario, we use an off-shelf single board computer equipped with a camera and band-pass infrared filter as a localization anchor, custom-built battery-powered smart LEDs as tags, and an industry standard low power wireless mesh communication infrastructure. OSLo also provides a practical way to calibrates the optical sensor using a world origin. Evaluation results demonstrate the viability, performance, and usability of OSLo.

\section{A. Optical Sensor}

We use Raspberry Pi Zero equipped with a Pi NoIR Camera as an optical sensor. Additionally, we attach a $940 \mathrm{~nm}+/-10 \mathrm{~nm}$ band-pass IR filter to the camera. All the components reside in a custom-designed 3D printed case. The combination of the NoIR camera with the band-pass IR provides numerous benefits. Since indoor environments do not commonly have IR radiation, the image formed on the camera is mostly blacked out. This blacked-out image greatly reduces the processing requirement for background subtraction during the first phase of image processing. Additionally, since it is impossible to identify people in the blacked-out image, the rendered images are privacy-preserving and therefore enable the system to be deployed even in environments with strong privacy requirements. Since the LED tags operate in the $940 \mathrm{~nm}$ range, the band-pass IR filter captures maximum radiant energy from the LED and creates a sharp image.

\section{Calibration}

The intrinsic and extrinsic parameters for each optical sensor are calculated separately in two steps, first, in the offline step the intrinsic calibration parameters are calculated using 
OpenCV library [24] function calibrateCamera() by providing multiple images of a 9x6 checkerboard pattern from various positions and angles. The extrinsic calibration parameters for each optical sensor are calculated after the optical sensor is installed at its deployment position. We first select a world origin point and fix the world coordinate system based on the chosen world origin point. Then we place 4 LED tags in the field of view of the optical sensor and manually measure the $\mathrm{x}, \mathrm{y}$, and $\mathrm{z}$ coordinates of the LED tags and provide the coordinates to the optical sensor along with the tag ids. As soon as the optical sensor detects the LED tags, it maps them to the provided LED tag world coordinates. The mapped LED tag coordinates along with the intrinsic parameters matrix are then supplied to the opencv [24] function CalibrateCamera2() to calculate the extrinsic parameters. Both intrinsic and extrinsic parameters are stored in the localization system and later used in localization using either the single-camera or multicamera based localization algorithms.

\section{B. Mesh Network}

The optical sensors were realised as plug-and-play peripherals for the VersaSense MicroPnP device [25], [26] and connected via a multi-hop IEEE 802.15.4 6LowPAN network using a commercial Time Synchronised Channel Hopping (TSCH) [27]. The use of a self-forming mesh network enables easy positioning of the optical sensors, each of which also serves as a router, thereby extending the range of the wireless network as deployment continues. The optical sensors also offer WiFi connections, which are used in the initial calibration process but thereafter deactivated to conserve resources.

Following the MicroPnP standard [28], all optical sensors were modeled as CoAP resources [29] . Alongside localization coordinate streams, the optical sensors expose state data including optical sensor power consumption and software state (powered off, crashed or running) along with controls to power cycle the device and turn WiFI on or off to support calibration and other maintenance activities.

All CoAP camera resources are automatically discovered and observed by the network gateway and exposed to the data processing back end via Message Queue Telemetry Transport (MQTT) and Web Sockets (WS) streams. In the back end, we use the TICK stack (Telegraf, InfluxDB, Chronograf, and Kapacitor) [30] for data storage, alerting and visualization.

\section{LED Localization Tag}

The LED localization tag is built using off the shelf components. The main circuit is designed around an AtMega 1284p [31] microcontroller that drives the optical transmission via an IR LED. To achieve the target manufacturing cost of $\$ 1$, the tag hardware is designed to be as simple as possible. The tag is powered by 3 off-the-shelf AA batteries connected in series. The total price of the Bill of Material (BoM) is broken down in Table II for quatities of 10k units (Price verified in June 2020 using https://octopart.com/. A full list of BoM and PCB design files can be found at [32].
TABLE II: Bill of material price break-down for LED Tag @10k order

\begin{tabular}{|c|c|c|}
\hline Components & Price (\$) & Percentage \\
\hline LED + driver circuit & 0.52 & $53.4 \%$ \\
Passive elements & 0.084 & $8.6 \%$ \\
Microcontroller & 0.026 & $2.7 \%$ \\
Battery & 0.303 & $31.1 \%$ \\
Accessory & 0.04 & $4.1 \%$ \\
\hline Total & 0.973 & \\
\hline
\end{tabular}

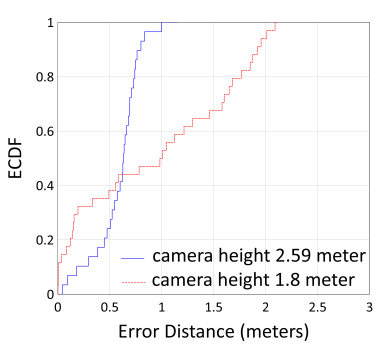

(a) Single Camera (b) Two Cameras

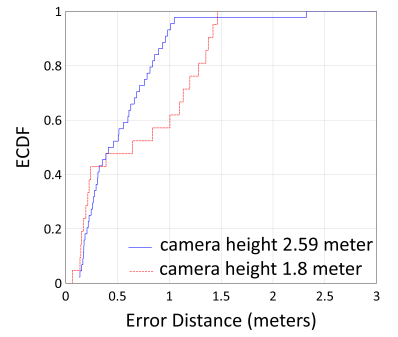

Fig. 5: Localization Error

\section{Evaluation}

We evaluate the OSLo localization system in an indoor environment. Our experimental evaluation focuses on localization accuracy with respect to the distance from the optical sensor (i.e. the camera). We empirically evaluated accuracy by placing the LED tag at uniformly distributed locations and measured the exact location with respect to the world origin. We then used OSLo to localize the observed LED tags. Finally, we calculated the localization error as the cartesian distance between the actual location and the location reported by OSLo.

\section{A. Physical Evaluation of Single Camera Localization}

We begin by evaluating single-camera localization accuracy at up to 35 meters from the camera. We placed the camera at two different heights to also test the effect of camera height on accuracy. Figure 5a shows an Empirical Cumulative Distribution Function (ECDF) of localization error by considering two different settings. The first one (blue solid line) is generated by placing the camera at a height of 2.59 meters. In this case, the observed localization error remains below 1 meter until the longest distance considered, 35 meters. The second configuration (red dotted line) shows the ECDF generated by placing the camera at a height of 1.8 meters. In this case, $50 \%$ of the observed data has an error of less than 1 meter at the longest distance, while the maximum error observed in this setting is slightly greater than 2 meters. As discussed in Section V-E, this behavior is explained by the steep angle between the camera and the LED plane.

\section{B. Physical Evaluation of Two Camera Localization}

We performed the same experiments mentioned in Section V-A leveraging the second OSLo variant, which employs two cameras as localization anchors. Figure $5 \mathrm{~b}$ shows the ECDF of 
localization error of the two different settings considered. The observed error is less than 1 meter until the $95^{\text {th }}$ percentile for the case when the cameras are at a 2.59-meter height. It is notable that the lower the camera height, the larger the localization error.

\section{Simulated Evaluation of Single Camera Localization}

In addition to the empirical evaluation, we evaluated the single camera-based localization algorithm using artificially generated pixel coordinates from real-world 3D points. The pixel coordinates were generated using projections of realworld coordinates on the image plane using the pinhole camera model in combination with real calibration parameters. Figure 6a shows the view frustum generated by the camera model. The simulated camera is mounted at a height of 10 meters and the view of the camera is set to an angle that covers a maximum distance of 35 meters. We generated 3D points within the view frustum of the camera, the ground truth image coordinates were calculated by projecting the $3 \mathrm{D}$ points to the image plane. A random error was introduced to the pixel coordinates to simulate tag detection errors based on Error $\hookrightarrow \mathcal{N}\left(\mu, \sigma^{2}\right)$, where $\mu=0$ and $\sigma=8$ was chosen. We then used the single-camera localization algorithm to calculate the 3D world coordinates from the image coordinates. The results are shown in Figures $6 b, 6 c, 6 d$ as calculated for the tag height of 0,2 and 4 meters respectively. The simulation shows that the single-camera localization algorithm gives less than 1 meter localization error.

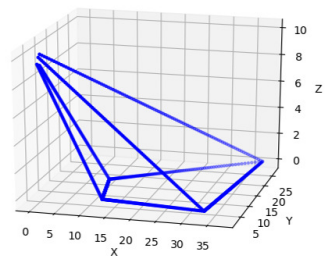

(a) View Frustum

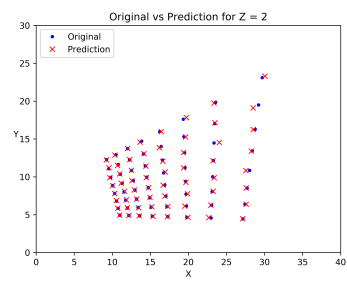

(c) Accuracy at $Z=2$

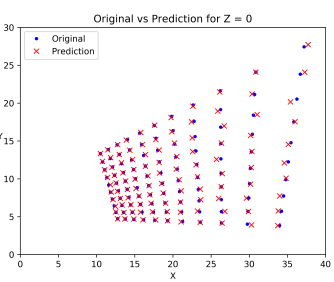

(b) Accuracy at $Z=0$

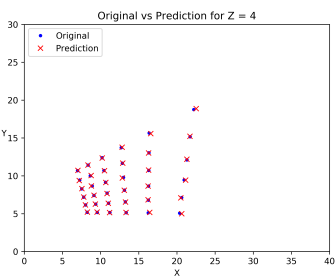

(d) Accuracy at $\mathrm{Z}=4$
Fig. 6: Single Camera-based Localization of Artificially generated 3D Points

\section{Simulated Evaluation of Two Camera Localization}

Similar to the single camera case, we evaluated the multiple camera-based localization algorithm using artificially generated image coordinates on two cameras. The two camera views were set in such a way that the field of view of the two cameras have an overlap in the 3D world space as shown in Figure $7 \mathrm{a}$ shows the view frustum generated by the camera model. We generated 3D points within the overlapping region of the view from the two cameras and calculated the pixel coordinates on image planes of the two cameras. We then introduced random error in pixel coordinates similar to the single camera case. Finally we calculated the 3D points based on the pixel coordinates from the two cameras. The results are shown in Figures 7b, 7c, 7d against the 3D coordinates on the plane $Z=0, Z=1$ and $Z=2$ respectively. The simulation for the two camera localization shows that the error is less than 1 meter for all simulated points.

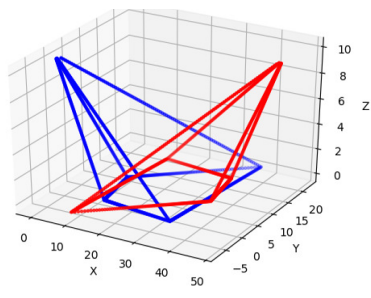

(a) View Frustum

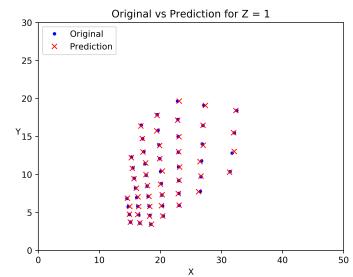

(c) Accuracy at $Z=1$

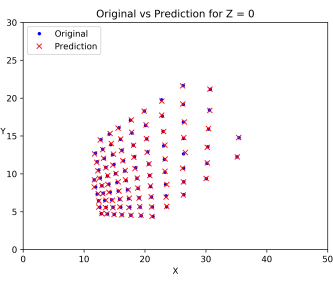

(b) Accuracy at $\mathrm{Z}=0$

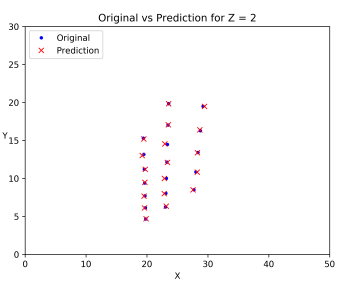

(d) Accuracy at $Z=2$
Fig. 7: Multiple Camera-based Localization of Artificially generated 3D Points

\section{E. Sources of Error}

In the remainder of this section, we highlight the primary causes of localization errors in OSLo.

Accuracy vs Distance: One of the main goals of OSLo is providing sub-meter accuracy at a long distance from the optical sensor. We therefore evaluated localization accuracy versus the distance from the optical sensor as shown in Figure 8a. The $\mathrm{X}$-axis represents the distance of the localization tag from the optical sensor, while the Y-axis represents the localization error, which is calculated as the cartesian distance between the location calculated by OSLo and the actual location. The figure illustrates that the localization error is a few centimeters when the distance considered is less than 8 meters, whereas it increases to not more than 1 meter at a distance of 35 meters. The error rises exponentially from a distance of 35 meters onward.

Observation Error: The pixel coordinates of the LED tag on the image are extracted by detecting blobs of connected pixels in the image after performing a background subtraction. Each blob is considered as a potential LED and the center of the blob is reported as its position. Due to fluctuation in 
(a) Localization Error vs Distance from Optical Sensor

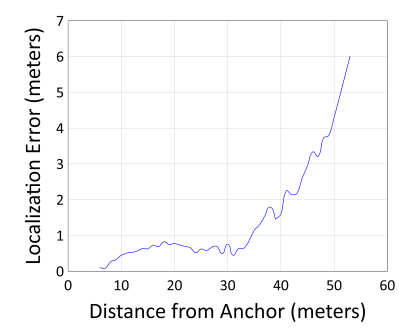

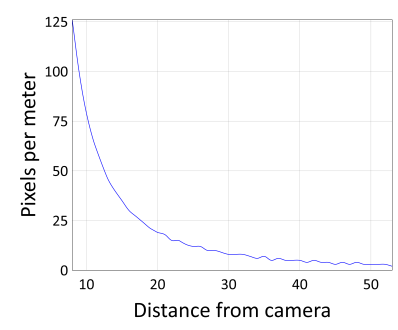

(b) Image Vs World Coordinates
Fig. 8: Localization Error

background luminance, it is possible that the centroid of the blob does not represent the true center of the LED. As a result, an error is introduced in the localization. We call this error an observation error.

Calibration Error: Both intrinsic and extrinsic camera calibrations may introduce errors as they are estimates based on image analysis. We observed that performing the intrinsic and extrinsic calibration independently can reduce the effects of calibration errors. Since the extrinsic calibration depends on the LED detection, it is also prone to observation error.

Reflections: Reflections are observed by the camera as separate LEDs. The camera cannot distinguish between a reflection and the real LED. However, the system can raise a warning when it observes the same LED at two locations simultaneously.

Distance from the Camera: As can be seen in Figure 8a, localization error remains under one meter until a distance of 35 meters but rises exponentially thereafter. This behavior can be understood by taking into account the perspective transformation of the target, which results in an image that represents nearby objects with more pixel density in comparison to faraway objects. As a result, the displacement of an object that is further from the camera is represented by fewer pixels than an object closer to the camera. Thus, the detection error of distant objects is higher compared to the nearby objects. We also observe this empirically as shown in Figure 8b, as the observation point moves away from the camera, the number of pixels on the image corresponding to one meter in the world decreases exponentially.

There are two limiting cases due to this effect. The first one is when the object is parallel to the camera. In other words, the object plane is parallel to the line of sight vector. In this case, the localization error will be infinite, as there is no single point of intersection between the LOS and the height plane. The other limiting case is that when the camera is pointing directly downwards, or the LOS is perpendicular to the object plane. In this case, the localization error will be minimal. However, in such a setting, the camera will cover very little area. Accordingly, a much more dense deployment of cameras will be needed. Keeping in view the two limiting cases, in an ideal deployment setting needs to utilize the maximum height available, while the orientation of the camera should be in such a way that the furthest point visible from the camera is at a distance where the pixel coverage of the localization tag is greater than observation error.

\section{F. LED Tag Power Consumption}

To evaluate the power consumption of the LED tag, we conducted an experiment wherein the tag transmitted on a short duty cycle interval of $50 \%$. The various specifications and configurations of the LED tag with respect to power consumption are illustrated in Table III. The tag transmitted 6352 localization messages before the battery was completely drained. The most significant factor that determines battery life is the number of transmitted localization messages. As a result, the maximum lifetime of the LED tag can be preset by adjusting the interval between localization messages. For example, if the tag updates its location by transmitting its ID every 90 minutes, 24 hours per day, 6352 messages would lead to a practical operating lifetime of 397 days with continuous localization updates. a smaller interval between the messages will result in a proportionally smaller operating lifetime.

TABLE III: Configurations used in evaluation

\begin{tabular}{|c|c|c|c|c|c|}
\hline $\begin{array}{c}\text { Sleep } \\
\text { Current }(\mathrm{uA})\end{array}$ & $\begin{array}{c}\text { MCU Active } \\
\text { Current }(\mathrm{mA})\end{array}$ & $\begin{array}{c}\text { LED Active } \\
\text { Current }(\mathrm{mA})\end{array}$ & $\begin{array}{c}\text { Duty } \\
\text { Cycle }(\%)\end{array}$ & $\begin{array}{c}\text { Packet } \\
\text { Size(bits })\end{array}$ & $\begin{array}{c}\text { Battery } \\
\text { Size }(\mathrm{Ah})\end{array}$ \\
\hline 2 & 8 & 1200 & 50 & 21 & 2.2 \\
\hline
\end{tabular}

\section{RELATED WORK}

Most state of the art localization systems aim at utilizing existing communication infrastructure for localization. These include WiFi access points [33]-[36] and other radio technologies such as FM Radio signals [37] and RFID [38]. Some radio-based localization techniques achieve better localization accuracy by modifying the WiFi access point [39]. Recent work on localization utilizes existing lighting infrastructure by modifying the LED luminaires to transmit their location information via visible light communication [10], [40], [41].

The approach presented in this paper is very different from the existing localization technologies. However, some techniques used in this paper can be related to parts of existing work. A component-level comparison between this paper and closely related existing work follows.

\section{A. Optical Localization}

Earlier research in indoor localization using optical communication and positioning showed that it is possible to achieve sub-meter accuracy by using ceiling-mounted LED lights as optical localization anchors [13]. In most cases, the ceilingmounted LEDs transmit their identification information using an on/off keying pattern. Such optical localization systems either use Photo Sensors or a Camera with the localization tag to receive the anchor ID. Photo Sensor-based localization tag to receive the optical signal from the photosensor, and calculate location based on the observed localization anchors [10], [42], [43]. The second type of localization system utilizes a camera as the localization tag. The camera utilizes its rolling shutter effect to receive high-frequency data, while computer 
vision-based techniques are used for location estimation [44], [45].

In contrast to the existing optical localization systems, OSLo uses a Camera as the localization anchor and an LED as the localization tag. This role flip provides an opportunity to make a low-cost stand-alone localization tag that only consists of an LED, a control circuit, and batteries. On the other hand, the localization anchor can have a coverage range of more than 30 meters, further reducing the cost of the overall system. To our knowledge, no such localization system exists that uses cameras as location anchor and an LED as the location tag.

\section{B. Data Transmission}

State of the art camera-based visible light communication utilizes the rolling shutter effect of the Complementary Metal Oxide Semiconductor (CMOS) sensor-based cameras to increase the data transfer rate [46]. The core requirement for using the rolling shutter effect is that the transmitter covers multiple pixel lines on the image in order to imprint light and dark bands on the image, which is then used to form a signal pattern. Large enough coverage of the image is only possible when the transmitter is at a short range and the transmitter itself has a large surface area. However, due to the long range of localization and small surface area of the transmitter, OSLo does not have the liberty of a large covered region on the image. Hence, The rolling shutter communication is not feasible in this work.

\section{CONCLUSION}

This paper presented the design, development, and evaluation of OSLo, an optical communication-based localization system. The presented system provides a practical localization solution for large indoor environments. The system can be deployed easily in existing industry settings. We identified and solved important problems that were required for industrial deployment of a low cost, low maintenance, long-range, and accurate localization system. Our evaluation results in indoor environments show sub-meter accuracy at a long distance from the anchor. Our work confirms the potential of using a camera as a localization anchor and a LED as the tag in a localization system.

By flipping the typical role of the anchor and tag, our approach opens the doors to further exploration of such techniques. More research is needed for configurations of the camera such as the use of a fish-eye lens, while the camera is pointing directly downwards. Both single camera and multiple camera-based configurations need to be explored to see their effect on localization accuracy and effective range of optical sensor. Furthermore, the current LED-based localization tags only have one-way communication. If a low power downstream channel is introduced in the LED-based tags, it will allow the transmission of data on-demand. This can potentially reduce localisation time and thereby increase the battery life of the LED greatly. Moreover, a downstream communication channel can be used to implement secure localization features to the system. Battery operated localization anchors will also increase the deployability of the system many fold. A Battery operated optical sensor will perform localization on-demand, instead of continuously monitoring the field of view. This will require a downstream channel to the LED tags. Additionally, it will require a deep sleep mode on the anchors along with a fast wake-up cycle.

\section{REFERENCES}

[1] Indoor location market size, share \& industry analysis report, 2023. (Accessed on 11/30/2020).

[2] Z. Chen, H. Zou, J. Yang, H. Jiang, and L. Xie. Wifi fingerprinting indoor localization using local feature-based deep 1stm. IEEE Systems Journal, 14(2):3001-3010, 2020.

[3] A. Mussina and S. Aubakirov. Rssi based bluetooth low energy indoor positioning. In 2018 IEEE 12th International Conference on Application of Information and Communication Technologies (AICT), pages 1-4, 2018.

[4] S. Lanzisera, D. Zats, and K. S. J. Pister. Radio frequency time-of-flight distance measurement for low-cost wireless sensor localization. IEEE Sensors Journal, 11(3):837-845, 2011.

[5] Deepak Vasisht, Swarun Kumar, and Dina Katabi. Decimeter-level localization with a single wifi access point. In 13th USENIX Symposium on Networked Systems Design and Implementation (NSDI 16), pages 165-178, Santa Clara, CA, March 2016. USENIX Association.

[6] B. GroBwindhager, M. Stocker, M. Rath, C. A. Boano, and K. Römer. Snaploc: An ultra-fast uwb-based indoor localization system for an unlimited number of tags. In 2019 18th ACM/IEEE International Conference on Information Processing in Sensor Networks (IPSN), pages 61-72, 2019.

[7] Nissanka B Priyantha, Anit Chakraborty, and Hari Balakrishnan. The cricket location-support system. In Proceedings of the 6th annual international conference on Mobile computing and networking, pages 32-43, 2000.

[8] Pengjin Xie, Lingkun Li, Jiliang Wang, and Yunhao Liu. Litag: localization and posture estimation with passive visible light tags. In Proceedings of the 18th Conference on Embedded Networked Sensor Systems, pages 123-135, 2020.

[9] Ye-Sheng Kuo, Pat Pannuto, Ko-Jen Hsiao, and Prabal Dutta. Luxapose: Indoor positioning with mobile phones and visible light. In Proceedings of the 20th Annual International Conference on Mobile Computing and Networking, MobiCom '14, page 447-458, New York, NY, USA, 2014. Association for Computing Machinery.

[10] Liqun Li, Pan Hu, Chunyi Peng, Guobin Shen, and Feng Zhao. Epsilon: A visible light based positioning system. In 11th \{USENIX\} Symposium on Networked Systems Design and Implementation ( $\{N S D I\}$ 14), pages 331-343, 2014

[11] Chi Zhang and Xinyu Zhang. Litell: Robust indoor localization using unmodified light fixtures. In Proceedings of the 22nd Annual International Conference on Mobile Computing and Networking, MobiCom '16, page 230-242, New York, NY, USA, 2016. Association for Computing Machinery.

[12] V. Walter, N. Staub, A. Franchi, and M. Saska. Uvdar system for visual relative localization with application to leader-follower formations of multirotor uavs. IEEE Robotics and Automation Letters, 4(3):26372644, 2019.

[13] Jean Armstrong, Y Ahmet Sekercioglu, and Adrian Neild. Visible light positioning: a roadmap for international standardization. IEEE Communications Magazine, 51(12):68-73, 2013.

[14] Soo-Yong Jung, Swook Hann, and Chang-Soo Park. Tdoa-based optical wireless indoor localization using led ceiling lamps. IEEE Transactions on Consumer Electronics, 57(4):1592-1597, 2011.

[15] Seongsu Lee and Sung-Yoon Jung. Location awareness using angleof-arrival based circular-pd-array for visible light communication. In 2012 18th Asia-Pacific Conference on Communications (APCC), pages 480-485. IEEE, 2012.

[16] Yohei Nakazawa, Hideo Makino, Kentaro Nishimori, Daisuke Wakatsuki, and Hideki Komagata. Indoor positioning using a high-speed, fish-eye lens-equipped camera in visible light communication. In International Conference on Indoor Positioning and Indoor Navigation, pages 1-8. IEEE, 2013. 
[17] Toshiya Tanaka and Shinichro Haruyama. New position detection method using image sensor and visible light leds. In 2009 Second International Conference on Machine Vision, pages 150-153. IEEE, 2009.

[18] Aaron Ganick and Daniel Ryan. Self identifying modulated light source, October 21 2014. US Patent 8,866,391.

[19] H Komagata, I Ishii, A Takahashi, D Wakatsuki, and H Imai. A geometric calibration method of internal camera parameter for fish-eye lenses. IEICE Trans. Information and Systems, 89:2006-1, 2006.

[20] Andrew Glassner. Graphics gems i. 1990.

[21] Richard Hartley and Andrew Zisserman. Multiple view geometry in computer vision. Cambridge university press, 2003.

[22] Adrian Bradski. Learning OpenCV, [Computer Vision with OpenCV Library ; software that sees]. O'Reilly Media, 1. ed. edition, 2008.

[23] Peter F Zalud and Robert M Evans. Manchester encoding and decoding system, May 16 2000. US Patent 6,064,705.

[24] G. Bradski. The OpenCV Library. Dr. Dobb's Journal of Software Tools, 2000.

[25] Versasense micropnp development starter kit - digikey. https://www. digikey.be/nl/blog/versasense-micropnp-development-starter-kit. (Accessed on 12/15/2020).

[26] Wilfried Daniels, Fan Yang, Nelson Matthys, Wouter Joosen, and Danny Hughes. Enabling plug-and-play for the internet of things. In Proceedings of the Posters and Demos Session of the 16th International Middleware Conference, pages 1-2, 2015.

[27] Thomas Watteyne, Lance Doherty, Jonathan Simon, and Kris Pister. Technical overview of smartmesh ip. In 2013 Seventh International Conference on Innovative Mobile and Internet Services in Ubiquitous Computing, pages 547-551. IEEE, 2013.

[28] Fan Yang, Nelson Matthys, Rafael Bachiller, Sam Michiels, Wouter Joosen, and Danny Hughes. $\mu$ pnp: Plug and play peripherals for the internet of things. In Proceedings of the Tenth European Conference on Computer Systems, EuroSys '15, New York, NY, USA, 2015. Association for Computing Machinery.

[29] Zach Shelby, Klaus Hartke, Carsten Bormann, and B Frank. Rfc 7252 The constrained application protocol (coap). Internet Engineering Task Force, 2014

[30] Influxdb 1.x: Open source time series platform - influxdata. https: //www.influxdata.com/time-series-platform/. (Accessed on 12/11/2020).

[31] Atmega1284p - 8-bit avr microcontrollers. https://www.microchip.com/ wwwproducts/en/ATMEGA1284P. (Accessed on 12/31/2020).

[32] Ford led - google sheets. https://docs.google.com/spreadsheets/d/ 1oMA2inNAM4bQxRCVzhOHOgB1rj32mcejxlMUecD10_g/edit\#gid= 0 . (Accessed on 12/15/2020).

[33] Zheng Yang, Zimu Zhou, and Yunhao Liu. From rssi to csi: Indoor localization via channel response. ACM Computing Surveys (CSUR), 46(2):1-32, 2013.

[34] Andrew M Ladd, Kostas E Bekris, Algis Rudys, Lydia E Kavraki, and Dan S Wallach. Robotics-based location sensing using wireless ethernet. Wireless Networks, 11(1-2):189-204, 2005.

[35] Jiang Xiao, Kaishun Wu, Youwen Yi, Lu Wang, and Lionel M Ni. Pilot: Passive device-free indoor localization using channel state information. In 2013 IEEE 33rd International Conference on Distributed Computing Systems, pages 236-245. IEEE, 2013.

[36] Praveen Kumar, Lohith Reddy, and Shirshu Varma. Distance measurement and error estimation scheme for rssi based localization in wireless sensor networks. In 2009 Fifth international conference on wireless communication and sensor networks (WCSN), pages 1-4. IEEE, 2009.

[37] Yin Chen, Dimitrios Lymberopoulos, Jie Liu, and Bodhi Priyantha. Fmbased indoor localization. In Proceedings of the 10th international conference on Mobile systems, applications, and services, pages 169182, 2012

[38] Lionel M Ni, Yunhao Liu, Yiu Cho Lau, and Abhishek P Patil. Landmarc: indoor location sensing using active rfid. In Proceedings of the First IEEE International Conference on Pervasive Computing and Communications, 2003.(PerCom 2003)., pages 407-415. IEEE, 2003.

[39] Jie Xiong and Kyle Jamieson. Arraytrack: A fine-grained indoor location system. In Presented as part of the 10th $\{$ USENIX $\}$ Symposium on Networked Systems Design and Implementation ( $\{N S D I\} 13)$, pages 7184, 2013.

[40] Saliha Büyükçorak and Güneş Karabulut Kurt. A bayesian perspective on rss based localization for visible light communication with heterogeneous networks extension. IEEE Access, 5:17487-17500, 2017.
[41] Yousef Almadani, Muhammad Ijaz, Sujan Rajbhandari, Umar Raza, and Bamidele Adebisi. Applications of visible light communication for distance estimation: a short survey. In 2019 IEEE Jordan International Joint Conference on Electrical Engineering and Information Technology (JEEIT), pages 261-265. IEEE, 2019.

[42] Chi Zhang and Xinyu Zhang. Pulsar: Towards ubiquitous visible light localization. In Proceedings of the 23rd Annual International Conference on Mobile Computing and Networking, pages 208-221, 2017.

[43] Yiqing Hu, Yan Xiong, Wenchao Huang, Xiang-Yang Li, Panlong Yang, Yanan Zhang, and Xufei Mao. Lightitude: Indoor positioning using uneven light intensity distribution. Proceedings of the ACM on Interactive, Mobile, Wearable and Ubiquitous Technologies, 2(2):1-25, 2018.

[44] Ye-Sheng Kuo, Pat Pannuto, Ko-Jen Hsiao, and Prabal Dutta. Luxapose: Indoor positioning with mobile phones and visible light. In Proceedings of the 20th annual international conference on Mobile computing and networking, pages 447-458, 2014.

[45] De Xu, Liwei Han, Min Tan, and You Fu Li. Ceiling-based visual positioning for an indoor mobile robot with monocular vision. IEEE Transactions on Industrial Electronics, 56(5):1617-1628, 2009.

[46] Hongbang Zhang and Fan Yang. Push the limit of light-to-camera communication. IEEE Access, 8:55969-55979, 2020. 